\title{
Association of Poverty Income Ratio with Physical Functioning in a Cohort of Patients with Systemic Lupus Erythematosus
}

\author{
Courtney Hoge $\left(\mathbb{D}\right.$, C. Barrett Bowling $(\mathbb{D})$, S. Sam Lim ${ }^{(\mathbb{D})}$, Cristina Drenkard $(\mathbb{D}$, \\ and Laura C. Plantinga ${ }^{\mathbb{D}}$
}

\begin{abstract}
Objective. To examine the association of income relative to the poverty threshold [poverty income ratio (PIR)] with self-reported physical functioning (PF) in a cohort of patients with systemic lupus erythematosus.

Methods. We used cross-sectional data on 744 participants from Georgians Organized Against Lupus (GOAL), and secondary analyses used data on 56 participants from a nested pilot study. Primary analyses used multivariable linear regression to estimate the association between PIR (categorized as $<1.00,1.00-1.99,2.00-3.99$, and $\geq 4.00$; lower PIR indicate higher poverty) and PF (scaled subscore from the Medical Outcomes Study Short Form-12 survey; range 0-100, higher scores indicate better functioning). Secondary analyses summarized complementary measures of PF as means or percentages by PIR (categorized as $<1.00,1.00-1.99$, and $\geq 2.00$ ).

Results. The mean age of participants was 48.0 years; $6.7 \%$ were male; $80.9 \%$ were black; and $37.5 \%, 21.0 \%, 29.6 \%$, and $12.0 \%$ had PIR of $<1.00,1.00-1.99,2.00-3.99$, and $\geq 4.00$, respectively. The overall mean PF score was 45.8 (36.2, 40.7, 55.5, and 61.2 for PIR of $<1.00,1.00-1.99$, 2.00-3.99, and $\geq 4.00)$. With adjustment, higher PIR remained associated with higher PF scores [2.00-3.99 vs $1.00-1.99: \beta=10.9$ (95\% CI $3.3-18.6)$; $\geq 4.00$ vs $1.00-1.99: \beta=16.2$ (95\% CI 6.4-26.0)]. In secondary analyses, higher PIR was also associated with higher scores for objective physical performance.

Conclusion. Our results show that higher income relative to the poverty threshold is associated with better PF across multiple domains, warranting further research into multicomponent functional assessments to develop individual treatment plans and potentially improve socioeconomic disparities in outcomes. (First Release May 15 2020; J Rheumatol 2020;47:983-90; doi:10.3899/jrheum.190991)
\end{abstract}

Key Indexing Terms:

SYSTEMIC LUPUS ERYTHEMATOSUS

Low socioeconomic status (SES) is an established risk factor for worse outcomes in patients with systemic lupus erythematosus (SLE), such as greater disease damage and

From the Department of Epidemiology, Rollins School of Public Health, Emory University, Atlanta, Georgia; Durham Veterans Affairs Geriatric Research Education and Clinical Center, Durham Veterans Affairs Medical Center (VAMC), and Department of Medicine, Duke University, Durham, North Carolina; and Department of Medicine, Emory University, Atlanta, Georgia, USA.

Supported in part by Public Health Service Grant UL1TR000454 from the Clinical and Translational Science Award Program, US National Institutes of Health, National Center for Advancing Translational Sciences. The GOAL cohort study is supported by the US Centers for Disease Control and Prevention Grant 1U01DP005119.

C. Hoge, MSPH, Department of Epidemiology, Rollins School of Public Health, Emory University; C.B. Bowling, MD, MSPH, Durham Veterans Affairs Geriatric Research Education and Clinical Center, Durham VAMC; S.S. Lim, MD, MPH, Department of Medicine, Emory University; C. Drenkard, MD, PhD, Department of Medicine, Emory University; L.C. Plantinga, PhD, Department of Epidemiology, Rollins School of Public Health, Department of Medicine, Emory University.

Address correspondence to L.C. Plantinga, Division of General Medicine and Geriatrics, Department of Medicine, Emory University, 1841 Clifton Road NE, Wesley Woods Health Center \#552, Atlanta, Georgia 30329, USA.E-mail: laura.plantinga@emory.edu

Accepted for publication February 10, 2020.

\section{QUALITY OF LIFE $\quad$ EPIDEMIOLOGY}

worse depressive symptomatology $y^{1,2,3,4,5,6,7}$. Additionally, patients with SLE often experience work loss ${ }^{8,9}$, activity limitations ${ }^{10}$, and reduced health-related quality of life (HRQOL) $)^{11,12,13,14,15,16}$, and low SES has been associated with lower HRQOL among patients with $\mathrm{SLE}^{5,7,17}$. In particular, studies examining HRQOL and SES have shown that lower individual-level and neighborhood-level SES have been associated with poorer physical functioning (PF) among patients with $\mathrm{SLE}^{5,7,17}$. To our knowledge, no study has used the income-to-poverty ratio, commonly known as the poverty income ratio (PIR), with more than 2 categories. The PIR, the official poverty measure of the US Census ${ }^{18}$, not only reflects individual SES relative to the poverty threshold but also accounts for household size, resulting in an estimate that provides a more accurate picture of an individual's poverty experience. For example, many patients with SLE may have no income because they are dependents, or they may have household members who act as full-time caretakers; whether these patients are "living in poverty" depends on the combined income of their entire household and number of household members depending on that income. Further, it may be important to use more than 2 PIR

Personal non-commercial use only. The Journal of Rheumatology Copyright () 2020. All rights reserved. 
categories, because the experience of living just above the poverty threshold likely differs substantially from the experience of living far above the threshold.

Studies of HRQOL have targeted mostly white populations ${ }^{5,6,7}$, despite black individuals having a greater burden of disease (e.g., black women have $>3$ times greater incidence of SLE than white women) ${ }^{19}$ and greater susceptibility for worse SLE-related outcomes ${ }^{1,3}$. We hypothesized that lower PIR would be associated with worse functioning among patients with SLE. Using cross-sectional data from the Georgians Organized Against Lupus (GOAL) cohort, an ongoing, population-based cohort predominantly comprising black participants, we examined the association between multiple categories of PIR and self-reported PF and whether the association differed by work status or race. In secondary analyses, using data from a nested ancillary pilot study, we also examined whether associations of PIR with functioning were consistent across a comprehensive set of measures related to $\mathrm{PF}$, including objective measures of physical performance.

\section{MATERIALS AND METHODS}

Study populations and data sources. For primary analyses, we used data from the ongoing GOAL cohort study, a population-based sample of patients with SLE from metropolitan Atlanta, Georgia. Recruitment and data collection methods have been published ${ }^{20}$. Briefly, participants of GOAL were primarily recruited from the existing Georgia Lupus Registry, a population-based registry funded by the US Centers for Disease Control and Prevention, which estimated the incidence and prevalence of SLE in metropolitan Atlanta ${ }^{19}$. Patients not included in the registry but who were receiving SLE treatment at Emory University, Grady Memorial Hospital (a large safety-net hospital in Atlanta), or from community rheumatologists in metropolitan Atlanta at the time of recruitment were recruited to enrich the cohort. Additionally, recruitment emphasized incident patients ( $\leq 2$ yrs since diagnosis) to minimize survival bias. All participants were recruited by mail, by telephone, or in person, with subsequent assessments performed annually since Wave 1 (baseline; September 2011-September 2012). A total of 850 participants who were aged $\geq 18$ years at the time of enrollment with a documented diagnosis of SLE [ $\geq 4$ revised American College of Rheumatology (ACR) criteria $^{21}$ or 3 ACR criteria with a final diagnosis of SLE by a board-certified rheumatologist] were included in Wave 1. We used a cross-sectional design to describe the association of PIR with measures of $\mathrm{PF}$, which were reported by questionnaire during a single wave of GOAL (Wave 5; June 2016-July 2017). There was a total of 814 adult participants in Wave 5 of GOAL. For primary analyses, participants were excluded if they were missing either the question comprising the PF summary score $(n=14)$, the PIR $(n=45)$, or any other covariates $(n=70)$, leaving 744 participants in the final models.

For secondary analyses, a cross-sectional design was used to examine the association of PIR with additional complementary measures of PF not identified in annual GOAL assessments (i.e., objective physical performance, reported activities of daily living, and history of falls), which were measured during study visits for a nested, GOAL-ancillary pilot study (October 2016-April 2017). Recruitment and data collection methods for the pilot have been described ${ }^{22}$. There were 60 participants in the pilot, and we excluded individuals missing information on PIR $(n=4)$ from analyses, yielding a sample of 56 participants.

The Emory University Institutional Review Board approved the main and ancillary study protocols (no. IRB00003656), and all participants provided informed consent.
Study variables. Self-reported PIR was estimated as the ratio of a household income, as reported by the participant, to the appropriate poverty threshold for household size $^{23}$, as defined by the US Census Bureau. PIR was grouped into categories of $<1.00,1.00-1.99,2.00-3.99$, and $\geq 4.00$ for primary analyses. When examining the association of PIR with complementary measures of PF among the $n=56$ included in these analyses, PIR was collapsed into categories of $<1.00$ (household income below the poverty threshold), 1.00-1.99, and $\geq 2.00$ (household income more than twice the poverty threshold) to maximize study power.

Details of PF. Self-reported PF, the primary outcome of interest, was ascertained from the self-administered Medical Outcomes Study Short Form-12 questionnaire (SF-12), a 12-item version of the SF-36 that is validated ${ }^{24}$ and recommended for use in $\mathrm{SLE}^{12}$. Scores for the PF subscale were calculated from responses to 2 items of the SF-12: "Does your health now limit you in moderate activities, such as moving a table, pushing a vacuum cleaner, bowling, or playing golf?" and "Does your health now limit you in climbing several flights of stairs?", with possible responses for both items of "Yes, limited a lot," "Yes, limited a little," and "No, not limited at all." The PF score was scaled 0-100, where higher scores represent better functioning ${ }^{25}$. In sensitivity analyses, PF was dichotomized as "Limited a lot" vs "Not limited a lot" for each question within the scaled PF subscore.

Complementary measures of PF in nested pilot. Physical performance was assessed using the Short Physical Performance Battery (SPPB) ${ }^{26}$. The SPPB assessed balance (ability to hold standing poses in different foot positions), gait speed (fastest of two 4-m walks at regular pace), and lower body strength (time taken to complete 5 chair stands without using arms), which were scored 0-4 (higher scores indicating better levels of physical performance). The physical performance score was the sum of these 3 individual scores (range $0-12)^{26}$.

Activities of daily living. Instrumental activities of daily living (IADL; e.g., food preparation and housework $)^{27}$ and basic activities of daily living (BADL; e.g., bathing and dressing $)^{28}$ were self-reported, yielding scores that were dichotomized as the ability to perform the activity independently or with minimal assistance versus the inability to perform the activity without assistance.

Falls. Participants were asked if they had fallen in the past year and how many falls they had had in the past year.

Other variables. All other variables were obtained with the Wave 5 GOAL questionnaires. SLE-related organ damage was assessed using the Self-Administered Brief Index of Lupus Damage (SA-BILD) score (range 0-30), where higher scores indicate greater levels of damage ${ }^{29,30}$ Depressive symptomatology was assessed with the 9-item Patient Health Questionnaire (PHQ-9; range 0-27), where higher scores indicate more severe depression symptomatology ${ }^{31}$. Current SLE activity was assessed using the Systemic Lupus Activity Questionnaire (SLAQ; range 0-44), with higher scores indicating greater SLE-related disease activity ${ }^{32}$. Participants self-reported their age at SLE onset, sex, race, ethnicity, years of education, work status, marital status, social support, and body mass index (BMI). Disease duration was calculated as the difference in age at survey and age at SLE onset.

Statistical analysis. Participant characteristics of GOAL were summarized overall and by PIR category using chi-square test, Fisher's exact test, ANOVA, or nonparametric equality of medians tests, as appropriate. For the association between PIR and PF scores, slopes $(\beta)$ and $95 \%$ CI were estimated with multivariable linear regression models. Adjustment was performed for age, race, sex, education, marital status, and disease duration, which were considered a priori confounders. Because SLE-related organ damage (SA-BILD), depression (PHQ-9), SLE-related disease activity (SLAQ), and BMI were considered potential mediators as well as confounders, separate adjustment for each of these factors was performed using the fully adjusted multivariable model. Interaction terms between PIR and race, current work status, and depression (PHQ-9 score $\geq 5$ vs $\leq$ 4) were included to assess potential effect modification by these variables.

Personal non-commercial use only. The Journal of Rheumatology Copyright @ 2020 . All rights reserved. 
To address the robustness of results to the scoring of PF, sensitivity analyses of the association between PIR and PF were performed using multivariable logistic regression models for each question of the PF subscore to estimate OR (95\% CI). Logistic regression analyses used an identical modeling strategy for linear regression models, but did not address effect modification. Complementary measures of physical performance were summarized overall and by PIR category. Scores for physical performance and self-reported functioning were reported as means or percentages, as appropriate. Comparisons of scores across PIR categories were tested through Fisher's exact test or nonparametric equality-of-means tests, as appropriate. All analyses were conducted using SAS version 9.4 (SAS), and the threshold for statistical significance was set at $\alpha=0.05$.

\section{RESULTS}

Characteristics of the SLE cohort. Overall, the mean age was 48.0 years, $6.7 \%$ were male, and $80.9 \%$ were black (Table 1). The prevalence of PIR $<1.00,1.00-1.99$, 2.00-3.99, and $\geq 4.00$ among GOAL participants was $37.5 \%, 21.0 \%, 29.6 \%$, and $12.0 \%$, respectively. Participants with lower PIR were younger, had lower educational attainment, were less likely to be married, and were more likely to be black. The mean ages at onset of SLE for PIR of < $1.00,1.00-1.99,2.00-3.99$, and $\geq 4.00$ were $30.1,34.3$, 32.6 , and 35.1 years, respectively. The mean years of disease duration at the time of the survey differed by PIR category, in that participants with a higher PIR were more likely to have longer disease duration. Both PHQ-9 and SLAQ scores differed significantly by PIR, in that participants with lower PIR were more likely to have higher depressive symptoms and disease activity scores. SA-BILD scores did not differ significantly by PIR category.

Association of PIR with self-reported PF in GOAL. The overall mean scaled PF score for GOAL participants was 45.8; and PF scores by PIR category, < 1.00, 1.00-1.99, 2.00-3.99, and $\geq 4.00$, were 36.2, 40.7, 55.5, and 61.2, respectively (Table 2). With adjustment for age, sex, and race, participants with a PIR $<1.00$ had a PF score that was, on average, 7.0 points lower than participants with a PIR of 1.00-1.99. Participants with a PIR of 2.00-3.99 had a mean PF score that was 13.1 points higher and participants with a PIR $\geq 4.00$ had a mean PF score 20.6 points higher than participants with a PIR of 1.00-1.99. Further adjustment for education, marital status, and disease duration did not substantially change these results. After multivariable adjustment and adjusting for SA-BILD, PHQ-9, and SLAQ

Table 1. Characteristics of patients with systemic lupus erythematosus participating in the Georgians Organized Against Lupus cohort (June 2016-July 2017) overall and categorized by poverty income ratio (PIR).

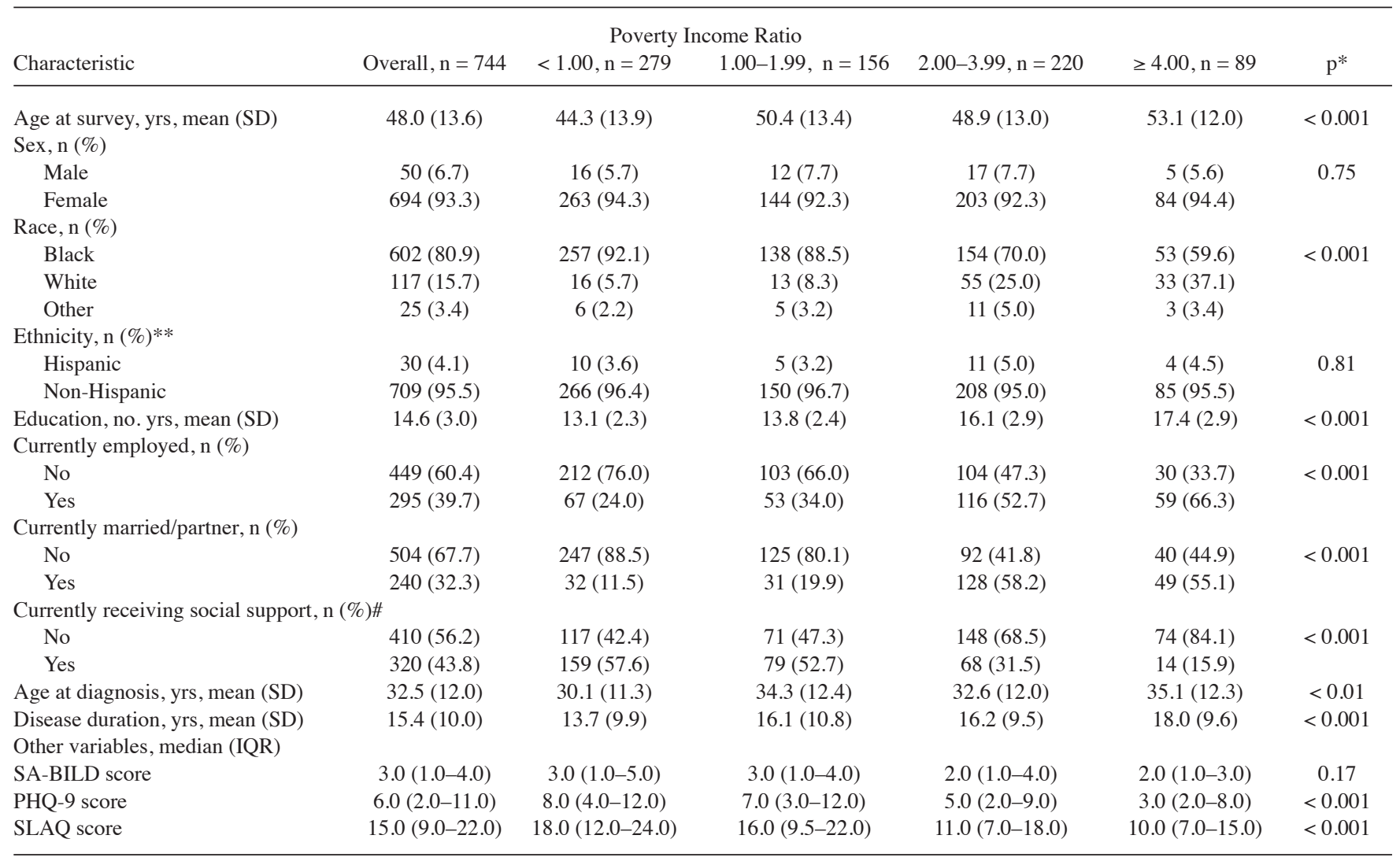

PIR is the ratio of household income to appropriate poverty threshold for household size, as defined by the U.S. Census Bureau (lower poverty income ratio indicates greater poverty). * By chi-square, Fisher's exact test, ANOVA, or nonparametric equality-of-medians test, as appropriate. $* *$ Missing data, $\mathrm{n}=739$. \# Missing data, $\mathrm{n}=730$. IQR: interquartile range; SA-BILD: Self-Administered Brief Index of Lupus Damage; PHQ-9: nine-item Patient Health Questionnaire; SLAQ: Systemic Lupus Activity Questionnaire: higher scores indicate more of the domain measured with these instruments.

Personal non-commercial use only. The Journal of Rheumatology Copyright $\subset$ 2020. All rights reserved. 
Table 2. Association between poverty income ratio (PIR) and self-reported physical functioning (PF) among participants with systemic lupus erythematosus in the Georgians Organized Against Lupus cohort (analysis of complete data, $\mathrm{n}=744$ ).

\begin{tabular}{|c|c|c|c|c|}
\hline \multirow[b]{2}{*}{ Outcome } & \multicolumn{3}{|c|}{ Poverty Income Ratio } & \multirow[b]{2}{*}{$\geq 4.00$} \\
\hline & $<1.00$ & $1.00-1.99$ & $2.00-3.99$ & \\
\hline PF score, mean $(\mathrm{SD}) *$ & $36.20(34.16)$ & $40.71(35.38)$ & $55.45(34.85)$ & $61.24(34.75)$ \\
\hline \multicolumn{5}{|l|}{ Difference in PF score $(95 \% \mathrm{CI})$} \\
\hline Unadjusted & $-4.50(-11.31,2.30)$ & 1.00 (ref) & $14.75(7.62,21.88)$ & $20.53(11.48,29.58)$ \\
\hline Adjusted for age, sex, and race & $-7.02(-13.79,-0.25)$ & 1.00 (ref) & $13.09(6.00,20.17)$ & $20.62(11.54,29.69)$ \\
\hline Multivariable-adjusted** & $-6.02(-12.81,0.76)$ & 1.00 (ref) & $10.90(3.25,18.55)$ & $16.21(6.39,26.03)$ \\
\hline Multivariable + SA-BILD score & $-4.13(-10.80,2.55)$ & 1.00 (ref) & $10.91(3.42,18.39)$ & $13.47(3.82,23.13)$ \\
\hline Multivariable + PHQ-9 score & $-4.92(-11.22,1.38)$ & 1.00 (ref) & $7.99(0.87,15.11)$ & $12.34(3.21,21.48)$ \\
\hline Multivariable + SLAQ score & $-2.56(-8.82,3.69)$ & 1.00 (ref) & $6.51(-0.55,13.57)$ & $9.17(0.08,18.25)$ \\
\hline Multivariable + BMI & $-7.27(-14.05,-0.49)$ & 1.00 (ref) & $10.51(2.88,18.13)$ & $16.02(6.18,25.87)$ \\
\hline
\end{tabular}

PIR is ratio of household income to appropriate poverty threshold for household size, as defined by the U.S. Census Bureau (lower PIR indicates greater poverty). * Scaled score of PF subscore from the 12-item Short Form Health Survey (scored 0-100, higher scores indicate better PF). **Adjusted for age, sex, race (black vs not black), education, marital status (married vs not married), and disease duration. SA-BILD: Self-Administered Brief Index of Lupus Damage; PHQ-9: nine-item Patient Health Questionnaire; SLAQ: Systemic Lupus Activity Questionnaire (higher scores indicate more of the domain measured with these instruments); BMI: body mass index.

scores individually, differences in PF scores by PIR were reduced; adjustment for SLAQ scores reduced differences in PF scores the most (Table 2). Interactions between PIR and work status, race, and depression were not statistically significant.

Sensitivity analyses. Sensitivity analyses in which the 2 SF-12 questions of the scaled PF score were dichotomized (Table 3) revealed comparable results to primary analyses. In comparison to participants with a PIR of $1.00-1.99$, participants with a PIR $<1.00$ had $26 \%$ increased likelihood of reporting that their health limited moderate activities, while participants with a PIR of 2.00-3.99 had 51\% reduced corresponding likelihood, and participants with a PIR $\geq 4.00 \mathrm{had}$
$64 \%$ reduced corresponding likelihood. Adjustment for age, sex, race, education, marital status, and disease duration did not considerably change these results. Similarly, individual adjustment of SA-BILD, PHQ-9, and SLAQ scores with multivariable adjustment did not substantially change the association. Participants with a PIR $<1.00$ were $30 \%$ more likely to report their health limiting their ability to climb several flights of stairs in comparison to those with a PIR of $1.00-1.99$, whereas those with a PIR of 2.00-3.99 were 54\% less likely and those with a PIR $\geq 4.00$ were $61 \%$ less likely to report limited ability to climb stairs. Further multivariable adjustment did not substantially change the association of PIR with individuals' health limiting their ability to climb

Table 3. Association between poverty income ratio (PIR) and low vs high physical functioning (PF) among participants with systemic lupus erythematosus in the Georgians Organized Against Lupus cohort: sensitivity analysis (analysis of complete data, $\mathrm{n}=744$ ).

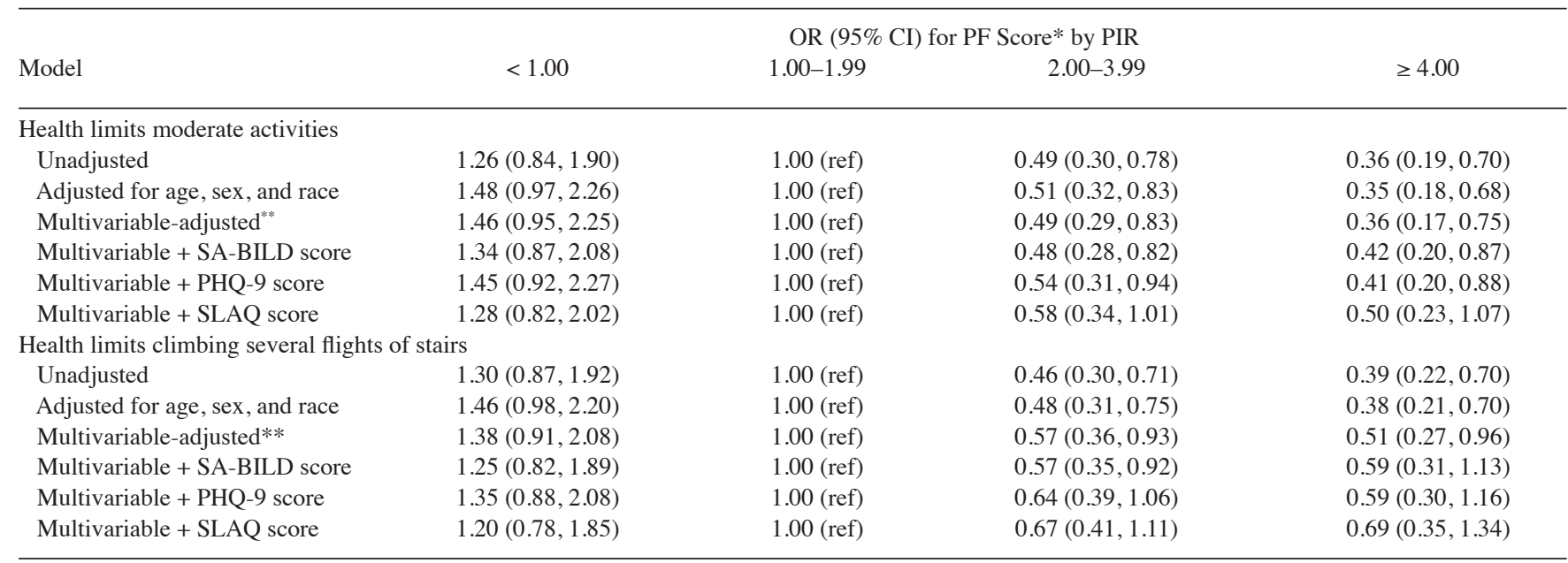

PIR is ratio of household income to appropriate poverty threshold for household size, as defined by the U.S. Census Bureau (lower PIR indicates greater poverty). * Dichotomized 2 questions comprising PF subscore from the 12-item Medical Outcomes Study Short Form (SF-12) health survey: limited a lot vs not limited a lot. ** Adjusted for age, sex, race (black vs not black), education, marital status (married vs not married), and disease duration. SA-BILD: SelfAdministered Brief Index of Lupus Damage; PHQ-9: nine-item Patient Health Questionnaire; SLAQ: Systemic Lupus Activity Questionnaire (higher scores indicate more of the domain measured with these instruments).

Personal non-commercial use only. The Journal of Rheumatology Copyright $\subset$ 2020. All rights reserved. 
several flights of stairs. Additional adjustment for SA-BILD, PHQ-9, SLAQ, and BMI scores separately gave similar estimates, but adjusting for SLAQ reduced differences in estimates closer to the null.

Complementary PF measures. The mean PF score for the nested pilot participants included in our study was 38.0, where participants with the highest PIR had the highest PF scores (Table 4). The overall mean balance score was 3.6, while the overall mean gait speed score was 3.4; however, neither balance nor gait speed scores differed statistically significantly by PIR category. The mean lower body strength scores for PIR of $<1.00,1.00-1.99$, and $\geq 2.00$ were 1.6, 1.4 , and 2.7 , respectively. For PIR $<1.00,1.00-1.99$, and $\geq 2.00$, the mean overall physical performance scores were 8.4, 8.2, and 10.2 . Overall, $35.7 \%$ of participants reported difficulty with food preparation, $14.3 \%$ reported difficulty with housework, $41.1 \%$ reported difficulty with shopping, and $12.5 \%$ reported difficulty with transportation; yet the only IADL that differed statistically significantly by PIR was transportation, where $22.6 \%$ of participants with a PIR
$<1.00$ reported difficulty with transportation and $0.0 \%$ of participants with PIR of $1.00-1.99$ and $\geq 2.00$ reported difficulty with transportation. Overall, $19.6 \%$ of pilot participants included in our study reported difficulty with incontinence, which was the only BADL that differed statistically significantly by PIR: $25.8 \%$ of participants with a PIR $<1.00$ reported difficulty with incontinence, $30.0 \%$ with a PIR of $1.00-1.99$ reported difficulty with incontinence, and $0.0 \%$ with a PIR $\geq 2.00$ reported difficulty with incontinence. The mean number of falls that participants reported in the year previous to the study was 2.1 , and falls were less frequently reported among those with a PIR $>2.00$ (26.7\% vs $48.4 \%$ and $70.0 \%$ for PIR $<1.00$ and $1.00-1.99$, respectively; Table 4 ).

\section{DISCUSSION}

In our study, self-reported PF scores were fairly low in a predominantly black cohort of individuals with SLE (the GOAL study), regardless of PIR category. The overall PF scores were below the mean of the healthy population in which the SF-12 was developed $(50.0)^{33,34}$, similar to previous

Table 4. Physical performance and self-reported function overall and categorized by poverty income ratio (PIR) among participants with systemic lupus erythematosus in the Georgians Organized Against Lupus ancillary pilot study (October 2016-April 2017). Higher scores reflect better functioning for all scales.

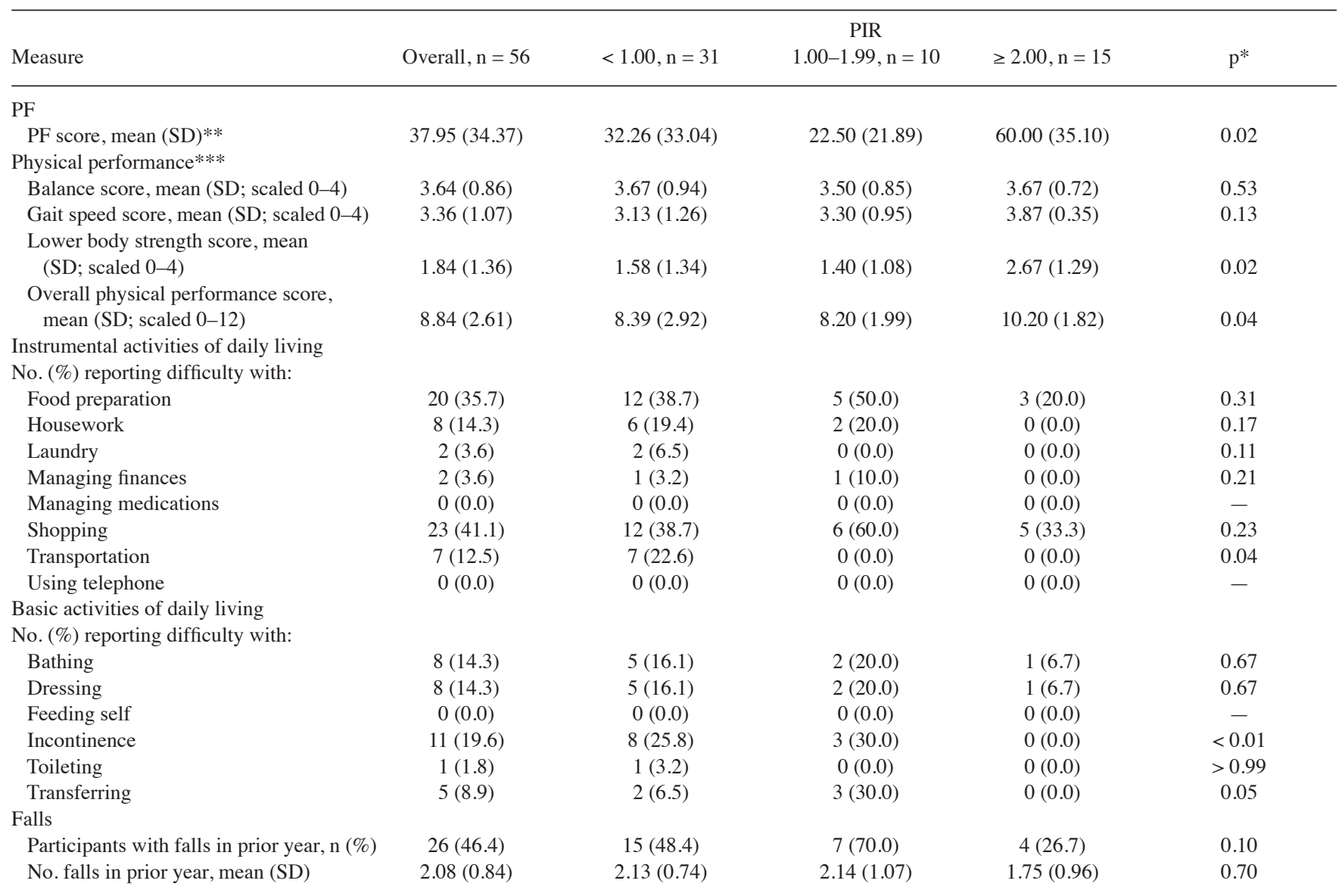

* Fisher's exact test or nonparametric equality-of-means test, as appropriate. ** Scaled score of PF subscore from the 12-item Medical Outcomes Study Short Form (health survey): scored 0-100, higher scores indicate better PF. ***Assessed with the Short Physical Performance Battery ${ }^{26}$. PF: physical functioning.

Personal non-commercial use only. The Journal of Rheumatology Copyright $\subset$ 2020. All rights reserved. 
studies investigating predictors of PF that also show lower $\mathrm{PF}$ scores for individuals with $\mathrm{SLE}^{35}$. On average, participants with higher PIR had higher PF scores in this study. However, differences between PF scores by PIR category were greatest among participants with the highest income relative to poverty level, compared to those at or just above the poverty threshold; whereas those with income below the poverty level had scores similar to those with income at the poverty level.

Participants of the nested pilot were demographically and clinically similar to the overall cohort ${ }^{22}$, and while those in the nested pilot had lower PF scores than those in the overall cohort (38.0 vs 45.8), associations of complementary measures of PF with PIR in the pilot, on average, reflected associations similar to those observed in the overall GOAL cohort. Although PIR of 2.00-3.99 and $\geq 4.00$ were collapsed into a single category for the nested pilot, the lowest and highest PIR categories of the pilot had PF scores similar to the lowest and highest PIR categories of GOAL (36.2 and 61.2 vs 32.3 and 60.0), indicating that levels of PF are not substantially different from the overall cohort from which participants were selected. Differences in physical performance scores, on average, were larger with higher PIR. Of the instrumental activities of daily living (IADL), a greater proportion of individuals with lower versus higher PIR reported difficulties with food preparation, housework, laundry, shopping, and transportation. Other IADL domains showed similar patterns, although they were not statistically significant. Statistically significant differences in the proportion of individuals reporting difficulties with BADL between PIR categories were observed only for incontinence, which was reported only among those at $(30 \%)$ or below $(26 \%)$ the poverty threshold.

Previous studies have shown that patients with SLE frequently have muscle weakness, high levels of fatigue, and low rates of physical activity ${ }^{36,37,38}$, resulting in reduced $\mathrm{PF}^{39,40,41}$, an important aspect of HRQOL. While perceived $\mathrm{PF}$ is important in addressing HRQOL, objective measures of PF have not been studied as thoroughly in SLE populations $^{22}$. Additionally, studies examining PF in SLE often do not use multicomponent assessments of functioning, such as those applied in populations of older adults. Measures of PF in older adults, such as IADL and BADL, history of falls, gait speed, and chair stands ${ }^{42}$, are predictors of worse mortality and health outcomes ${ }^{26}$. We found that multiple components of functioning may be associated with socioeconomic status (SES). Further, while lower individual-level and neighborhood-level SES have been shown to be associated with poorer PF among patients with SLE ${ }^{5,7,17}$, to our knowledge, none have used multiple categories of the income-to-poverty ratio, commonly known as the PIR, which provides a more in-depth measurement of relative poverty.

Because our method of determining PF scores ${ }^{25}$ is not validated across studies of HRQOL, it is unknown whether the estimated differences reflect clinically important differences in PF. However, using the statistical definition of a minimally important difference in PF scores as half an SD of the PF scores ${ }^{43}$ from the overall GOAL cohort (= 18.0 points), we found that the range of mean unadjusted $\mathrm{PF}$ scores was 36.2-61.2, indicating a minimally important difference in PF scores across all PIR categories by this definition. However, pairwise differences in mean unadjusted PF scores between adjacent PIR categories were not meaningful.

In the ancillary pilot study, substantial levels of impairment in physical performance and self-reported functioning were found, irrespective of PIR category. For many domains, increasing PIR was associated with less impairment; however, we also found slightly greater impairment among participants with a PIR of 1.00-1.99 than among those with a PIR of $<1.00$ for balance, lower body strength, and overall physical performance scores. Regardless of PIR, physical performance in this SLE cohort was comparable to, and sometimes lower than, that in the older adult population (age $\geq 70$ years) in which the test was developed ${ }^{26}$. In a population-based sample of older adults born before 1947 in the United States, adults who had more sources of income had faster gait speed ${ }^{44}$, which corresponds to the similar association of increased gait speed scores with higher PIR found in our study.

Our study has limitations and strengths worth mentioning. First, the study was cross-sectional, which limits causal inference, and the lack of longterm followup data means that we do not know individual trajectories in PIR or PF over time. Exclusions due to missing data, especially with regard to PIR, may have led to selection bias. Because PF scores were determined using 2 questions from the SF-12 survey, the measure may not adequately represent $\mathrm{PF}$, and misclassification may have occurred. Functioning may fluctuate over time with SLE activity, so a single measure of PF may not accurately portray participants' functioning. As with all observational studies, it is possible that we have not accounted for unknown confounders, and thus have residual confounding. Because our cohort was predominantly black, the study was likely inadequately powered to examine effect modification by race. For the complementary outcomes measured only in our nested pilot study, the small sample size further decreased the power to examine factors that influence, confound, or modify functioning. Generalizability of the results beyond metropolitan Atlanta may be limited, because the cohort is a population-based sample reflecting the demographics of this specific area.

Despite these limitations, our study has several strengths, such as the large sample size of GOAL. A population-based sample of patients with SLE with adequate representation of black individuals yields an accurate portrayal of HRQOL in a diverse cohort. Sensitivity analyses showing that the association between PIR and PF remained after dichotomizing

Personal non-commercial use only. The Journal of Rheumatology Copyright $@$ 2020 . All rights reserved. 
the outcome reduce concerns about whether the measurement of PF scores was too crude. Finally, the use of multidomain functional assessments is relatively novel in SLE populations, providing new insight that may allow for developing individual treatment plans and improving disparities in outcomes.

Lower income, relative to poverty thresholds and household size, may be associated with worse functioning across multiple domains in SLE. Given these results, future studies could include in-depth assessment of SES (taking relative poverty into account), multidomain functioning assessments in a larger cohort, and investigation of trajectories in both relative poverty and functioning. Further research into multicomponent functional assessments to develop individual treatment plans and potentially improve socioeconomic disparities in outcomes is warranted.

\section{ACKNOWLEDGMENT}

We thank the participants of GOAL. We also thank Benjamin Tift, Mechelle Lockhart, and Charmayne Dunlop-Thomas for invaluable assistance with recruitment and functioning assessments.

\section{REFERENCES}

1. Carter EE, Barr SG, Clarke AE. The global burden of SLE: prevalence, health disparities and socioeconomic impact. Nat Rev Rheumatol 2016;12:605-20.

2. Sule $\mathrm{S}$, Petri M. Socioeconomic status in systemic lupus erythematosus. Lupus 2006;15:720-3.

3. Drenkard C, Dunlop-Thomas C, Easley K, Bao G, Brady T, Lim SS. Benefits of a self-management program in low-income African-American women with systemic lupus erythematosus: results of a pilot test. Lupus 2012;21:1586-93.

4. Yelin E, Trupin L, Yazdany J. A prospective study of the impact of current poverty, history of poverty, and exiting poverty on accumulation of disease damage in systemic lupus erythematosus. Arthritis Rheum 2017;69:1612-22.

5. Trupin L, Tonner MC, Yazdany J, Julian LJ, Criswell LA, Katz PP, et al. The role of neighborhood and individual socioeconomic status in outcomes of systemic lupus erythematosus. J Rheumatol 2008;35:1782-8.

6. McCormick N, Trupin L, Yelin EH, Katz PP. Socioeconomic predictors of incident depression in systemic lupus erythematosus. Arthritis Care Res 2018;70:104-13.

7. Jolly M, Mikolaitis RA, Shakoor N, Fogg LF, Block JA. Education, zip code-based annualized household income, and health outcomes in patients with systemic lupus erythematosus. J Rheumatol 2010;37:1150-7

8. Baker K, Pope J, Fortin P, Silverman E, Peschken C. Work disability in systemic lupus erythematosus is prevalent and associated with socio-demographic and disease related factors. Lupus 2009;18:1281-8.

9. Drenkard C, Bao G, Dennis G, Kan HJ, Jhingran PM, Molta CT, et al. Burden of systemic lupus erythematosus on employment and work productivity: data from a large cohort in the southeastern United States. Arthritis Care Res 2014;66:878-87.

10. Björk M, Dahlström Ö, Wetterö J, Sjöwall C. Quality of life and acquired organ damage are intimately related to activity limitations in patients with systemic lupus erythematosus. BMC Musculoskelet Disord 2015;16:188.

11. McElhone K, Abbott J, Teh LS. A review of health related quality of life in systemic lupus erythematosus. Lupus 2006;15:633-43.
12. Kiani AN, Strand V, Fang H, Jaranilla J, Petri M. Predictors of self-reported health-related quality of life in systemic lupus erythematosus. Rheumatology 2013;52:1651-7.

13. Williams EM, Zhang J, Anderson J, Bruner L, Tumiel-Berhalter L. Social support and self-reported stress levels in a predominantly African American sample of women with systemic lupus erythematosus. Autoimmune Dis 2015;2015:40162.

14. Moldovan I, Katsaros E, Carr FN, Cooray D, Torralba K, Shinada $\mathrm{S}$, et al. The Patient Reported Outcomes in Lupus (PATROL) study: role of depression in health-related quality of life in a Southern California lupus cohort. Lupus 2011;20:1285-92.

15. Mazzoni D, Cicognani E, Prati G. Health-related quality of life in systemic lupus erythematosus: a longitudinal study on the impact of problematic support and self-efficacy. Lupus 2017;26:125-31.

16. Calderón J, Flores P, Aguirre JM, Valdivia G, Padilla O, Barra I, et al. Impact of cognitive impairment, depression, disease activity, and disease damage on quality of life in women with systemic lupus erythematosus. Scand J Rheumatol 2017;46:273-80.

17. Kulczycka L, Sysa-Jedrzejowska A, Zalewska-Janowska A, Miniszewska J, Robak E. Quality of life and socioeconomic factors in Polish patients with systemic lupus erythematosus. J Eur Acad Dermatol Venereol 2008;22:1218-26.

18. National Research Concil. Measuring poverty: a new approach. Citro CF, Michael RT, editors. Washington, DC: The National Academies Press; 1995. [Internet. Accessed April 24, 2020.] Available from: www.nap.edu/catalog/4759/ measuring-poverty-a-new-approach

19. Lim SS, Bayakly AR, Helmick CG, Gordon C, Easley KA, Drenkard C. The incidence and prevalence of systemic lupus erythematosus, 2002-2004: The Georgia Lupus Registry. Arthritis Rheum 2014;66:357-68.

20. Drenkard C, Rask KJ, Easley KA, Bao G, Lim SS. Primary preventive services in patients with systemic lupus erythematosus: study from a population-based sample in southeast U.S. Semin Arthritis Rheum 2013;43:209-16.

21. Hochberg MC. Updating the American College of Rheumatology revised criteria for the classification of systemic lupus erythematosus. Arthritis Rheum 1997;40:1725.

22. Plantinga L, Tift BD, Dunlop-Thomas C, Lim SS, Barrett Bowling C, Drenkard C. Geriatric assessment of physical and cognitive functioning in a diverse cohort of systemic lupus erythematosus patients: a pilot study. Arthritis Care Res 2018;70:1469-77.

23. US Census Bureau. Income, poverty, and health insurance coverage in the United States: 2011. Washington, DC. [Internet. Accessed April 24, 2020.] Available from: www.census.gov/newsroom/ releases/archives/income_wealth/cb12-172.html

24. Jenkinson C, Layte R, Jenkinson D, Lawrence K, Petersen S, Paice $\mathrm{C}$, et al. A shorter form health survey: can the SF-12 replicate results from the SF-36 in longitudinal studies? J Public Health 1997;19:179-86.

25. Ware J, Kosinski M, Turner-Bowker D, Gandek B. User's manual for the SF-12v2 health survey with a supplement documenting SF-12 health survey. Lincoln, RI: QualityMetric Inc.; 2002.

26. Guralnik JM, Simonsick EM, Ferrucci L, Glynn RJ, Berkman LF, Blazer DG, et al. A short physical performance battery assessing lower extremity function: association with self-reported disability and prediction of mortality and nursing home admission. J Gerontol 1994;49:M85-94.

27. Lawton MP, Brody EM. Assessment of older people: self-maintaining and instrumental activities of daily living. Gerontologist 1969;9:179-86.

28. Katz S, Downs TD, Cash HR, Grotz RC. Progress in development of the index of ADL. Gerontologist 1970;10:20-30.

29. Yazdany J, Trupin L, Gansky SA, Dall'Era M, Yelin EH, Criswell LA, et al. Brief index of lupus damage: a patient-reported measure

Personal non-commercial use only. The Journal of Rheumatology Copyright @ 2020 . All rights reserved. 
of damage in systemic lupus erythematosus. Arthritis Care Res 2011;63:1170-7.

30. Drenkard C, Yazdany J, Trupin L, Katz PP, Dunlop-Thomas C, Bao $\mathrm{G}$, et al. Validity of a self-administered version of the brief index of lupus damage in a predominantly African American systemic lupus erythematosus cohort. Arthritis Care Res 2014;66:888-96.

31. Kroenke K, Spitzer RL, Williams JB. The PHQ-9. J Gen Intern Med 2001;16:606-13.

32. Karlson EW, Daltroy LH, Rivest C, Ramsey-Goldman R, Wright EA, Partridge AJ, et al. Validation of a systemic lupus activity questionnaire (SLAQ) for population studies. Lupus 2003;12:280-6.

33. Ware JE, Kosinksi M, Keller SD. A 12-item short-form health survey: construction of scales and preliminary tests of reliability and validity. Med Care 1996;34:220-33.

34. McHorney CA, Ware JE Jr, Rogers W, Raczek AE, Lu JF. The validity and relative precision of MOS short- and long-form health status scales and Dartmouth COOP charts. Results from the Medical Outcomes Study. Med Care 1992;30 Suppl 5:MS253-65.

35. Devilliers H, Amoura Z, Besancenot JF, Bonnotte B, Pasquali JL, Wahl D, et al. Responsiveness of the 36-item Short Form Health Survey and the Lupus Quality of Life Questionnaire in SLE. Rheumatology 2014;54:940-9.

36. Mahieu MA, Ahn GE, Chmiel JS, Dunlop DD, Helenowski IB, Semanik P, et al. Fatigue, patient reported outcomes, and objective measurement of physical activity in systemic lupus erythematosus. Lupus 2016;25:1190-9.

37. Sterling KL, Gallop K, Swinburn P, Flood E, French A, Al Sawah $\mathrm{S}$, et al. Patient-reported fatigue and its impact on patients with systemic lupus erythematosus. Lupus 2014;23:124-32.
38. Andrews JS, Trupin L, Schmajuk G, Barton J, Margaretten M, Yazdany J, et al. Muscle strength, muscle mass, and physical disability in women with systemic lupus erythematosus. Arthritis Care Res 2015;67:120-7.

39. Thumboo J. Measuring functional status in patients with systemic lupus erythematosus. APLAR J Rheumatol 2003;6:184-7.

40. Piga M, Congia M, Gabba A, Figus F, Floris A, Mathieu A, et al. Musculoskeletal manifestations as determinants of quality of life impairment in patients with systemic lupus erythematosus. Lupus 2018;27:190-8.

41. Boström C, Dupré B, Tengvar P, Jansson E, Opava CH, Lundberg IE. Aerobic capacity correlates to self-assessed physical function but not to overall disease activity or organ damage in women with systemic lupus erythematosus with low-to-moderate disease activity and organ damage. Lupus 2008;17:100-4.

42. Brenowitz WD, Hubbard RA, Crane PK, Gray SL, Zaslavsky O, Larson EB. Longitudinal associations between self-rated health and performance-based physical function in a population-based cohort of older adults. PLoS One;2014;9:e111761.

43. Norman GR, Sloan JA, Wyrwich KW. Interpretation of changes in health-related quality of life: the remarkable universality of half a standard deviation. Med Care 2003;41:582-92.

44. Haas SA, Krueger PM, Rohlfsen L. Race/ethnic and nativity disparities in later life physical performance: the role of health and socioeconomic status over the life course. J Gerontol B Psychol Sci Soc Sci 2012;67:238-48. 